\title{
Comparación de la Evaluación Tradicional del Desarrollo Psicomotor versus una Prueba Autoadministrada
}

\author{
LUISA SCHONHAUT B. ${ }^{1}$, IVÁN ARMIJO R. ${ }^{2}$, TERESA MILLÁN K. ${ }^{3}$, JOSEFINA HERREROS A. ${ }^{4}$, \\ KATHERINE HERNÁNDEZ R. ${ }^{5}$, ANA MARÍA SALGADO V. ${ }^{6}$, MIGUEL ÁNGEL CORDERO V. ${ }^{7}$ \\ 1. Departamento de Pediatría, Facultad de Medicina Clínica Alemana - Universidad del Desarrollo. \\ 2. Escuela de Psicología Universidad del Desarrollo. \\ 3. Departamento de Pediatría Campus Occidente, Universidad de Chile. \\ 4. Consultora H\&B. \\ 5. Centro de Salud Familiar Los Quillayes-Corporación Municipal de La Florida. \\ 6. Centro de Salud Familiar Cristo Vive. \\ 7. Sistema de Protección Integral a la Infancia - Chile Crece Contigo, Ministerio de Salud de Chile.
}

\begin{abstract}
Comparison of Traditional Psychomotor Development Evaluation versus a Self-Administered Test?

Introduction: The purpose of this study is to evaluate the applicability of a self-administered questionnaire in the screening of psychomotor development $(\mathrm{PD})$ in a primary care setting $(\mathrm{PC})$. For that purpose, the degree of concordance between the Ages and Stages Questionnaire (ASQ) and a Psychomotor Development Evaluation Scale (Escala de Evaluación del Desarrollo Psicomotor-EEDP) currently utilized in PC was tested. In addition, perceptions and value judgment by professionals who participated in the study were explored. Methodology: This semi-quantitative study was performed between November 2008 and December 2009 in two PC centers of the Metropolitan Area of Chile on full-term newborns of the well-baby clinic. At age 8 and 18 months, parents or primary caretakers were asked to fill out the ASQ, and a PC professional completed the EEDP. Correlation and concordance between both tests was measured. Interviews and mini-focus groups were conducted with the participating professionals. Results: Three hundred and thirty newborns were recruited in the study. PD deficits were found on $8.79 \%$ of patients through the use of EEDP and in $12.73 \%$ through the use of ASQ $(p=0,05)$. Correlation between both evaluations was acceptable (r 0,5) as was concordance in deficit detection (kappa 0.576). The qualitative analysis showed appreciation of ASQ as a measuring tool as well as promoting awareness of PD. Staff of the PC settings utilized various strategies to overcome deficiencies of a self-administered test in populations of higher socio-educational and cultural risk. Conclusions: ASQ picked up significantly more PM deficits than EEDP, while correlation and concordance indices between both tests were adequate. Considering the subjective advantages expressed by staff in favor of ASQ, it is proposed that
\end{abstract}

Trabajo recibido el 19 de julio de 2010, devuelto para corregir el 07 de septiembre de 2010, segunda versión el 15 de septiembre de 2010, aceptado para publicación el 07 de octubre de 2010.

Financiamiento: Este proyecto fue financiado por el proyecto FONIS SA7i20043.

Correspondencia a:

Luisa Schonhaut B.

E-mail: Ischonhaut@alemana.cl 
those PC centers that wish to implement the ASQ do so by progressively incorporating parents and caretakers into the observation of their children's development.

(Key words: Screening, psicomotor development, Ages and Stages Questionnaires).

Rev Chil Pediatr 2010; 81 (6): 498-505

\section{RESUMEN}

Introducción: El propósito del estudio fue evaluar la aplicabilidad de un cuestionario de autoreporte para el screening del desarrollo psicomotor (DSM) en atención primaria de salud (APS). Para ello se analizó el grado de acuerdo entre el cuestionario Ages and Stages (ASQ) y la Escala de Evaluación del Desarrollo Psicomotor (EEDP), que es el método empleado de rutina en APS. Además, se exploraron las percepciones y valoraciones comparativas de profesionales que participaron en la aplicación de dichos test. Metodología: Estudio cuanticualitativo, realizado entre noviembre 2008 y diciembre 2009. En 2 centros de Salud de la Región Metropolitana de Chile se reclutó lactantes nacidos de término, que acudieron a control sano. A los 8 y 18 meses se les solicitó a los padres o cuidadores principales completar el ASQ y un profesional de APS aplicó el EEDP. Se midió la correlación y concordancia entre ambos test. Se aplicaron entrevistas individuales y mini focus groups a los profesionales participantes en las evaluaciones. Resultados: Fueron reclutados 330 lactantes. La frecuencia de déficit del DSM fue 8,79\% en EEDP y 12,73\% en ASQ (p0,05). Hubo correlación entre ambas evaluaciones ( $\mathrm{r} 0,5)$ y la concordancia en la pesquisa de déficit de DSM fue buena (kappa 0.576). En el análisis cualitativo destacó la valoración positiva del ASQ, tanto como instrumento de medición, como guía para promover el DSM. Para suplir las debilidades del ASQ, relacionadas con la modalidad de autoreporte, en grupos de mayor riesgo socio cultural o educacional, los centros de salud adoptaron diferentes estrategias. Conclusiones: El ASQ pesquisó significativamente más déficits del DSM que el EEDP, mientras que la correlación y concordancia entre ambas pruebas fueron adecuadas. Considerando las ventajas comparativas del ASQ en opinión de los profesionales, se propone que los centros de APS que deseen implementar ASQ, tomen una estrategia progresiva basada en la integración de los padres, madres y cuidadores en la observación del desarrollo de sus hijos.

(Palabras clave: Screening, desarrollo psicomotor, Ages and Stages Questionnaires).

Rev Chil Pediatr 2010; 81 (6): 498-505

\section{Introducción}

La evaluación del desarrollo psicomotor (DSM) es una actividad central en la supervisión de salud del lactante y el preescolar. Para lograr una oportuna pesquisa y derivación de los niños con rezago, diversas sociedades científicas recomiendan una vigilancia sistemática del DSM en los controles de salud y la aplicación de pruebas estandarizadas a edades específicas ${ }^{1}$. A nivel mundial existen distintas escalas validadas para este fin, cada una con sus propias particularidades, sin existir acuerdo general sobre cuál sería el mejor método.

En nuestro país, el Programa de Salud Infantil en la Atención Primaria de Salud (APS) contempla la evaluación y estimulación del DSM, a través de dos escalas que fueron desarrolladas en nuestro país y validadas en la década del 70 y 80: la Escala de Evaluación del Desarrollo Psicomotor (EEDP) $)^{2}$ a los 8 y 18 meses y el test de Desarrollo Psicomotor (TEPSI) a la edad de 3 años $^{3}$. Estos métodos realizan la evaluación y objetivación de logros del desarrollo a través de la observación directa del niño o niña por parte de una enfermera u otro profesional entrenado, en una sala de examen acondicionada y adecuadamente equipada.

Según datos del Ministerio de Salud 2005, la prevalencia de déficit del DSM en APS es de $6,6 \%$ a los 18 meses y $10,5 \%$ a los 4 años $^{4}$, tasa inferior a la esperada, de acuerdo a estadísticas internacionales ${ }^{5,6}$. Estudios recientes sugieren que estas escalas estarían subdiagnósticando niños con déficit en áreas específicas del desarrollo ${ }^{7}$. 
Por este motivo, en la Encuesta Nacional de Calidad de Vida y Salud se incorporó un método alternativo de medición del DSM, en que se preguntó a los padres o cuidador principal del niño, por el logro de las conductas esperadas para cada edad ${ }^{8}$. Esta encuesta mostró que cerca de un tercio de los niños menores de cinco años tenía déficit en su DSM 9 .

Las diferencias halladas entre estos dos métodos de evaluación, uno que mira directamente la conducta del niño en la sala de examen y otro que pregunta a los padres o cuidadores sobre lo que ellos observan de la conducta de sus hijos, ha llevado a plantear cuál de estos dos métodos puede ser más preciso en la detección de niños con riesgo de tener algún problema en su desarrollo.

En la última década han adquirido especial atención los cuestionarios autoadministrados, dado que son de bajo costo, fáciles de completar y no prolongan el tiempo dedicado a la consulta ${ }^{10,11}$. Uno de dichos test, el Ages \& Stages Questionnaires (ASQ) ${ }^{12}$, fue desarrollado en 1980 y recientemente reeditado. Este cuestionario es empleando ampliamente en Estados Unidos y en varios otros países en que ha sido validado $^{13-15}$.

En nuestro país se evidenció que el ASQ es un instrumento adecuado para el tamizaje del DSM en niños de nivel socio económico medio-alto ${ }^{16}$, no obstante, desconocemos cómo es el comportamiento y aplicabilidad en niños de nivel socio económico medio-bajo, y si su implementación presenta ventajas sobre los instrumentos que tradicionalmente se emplean en APS, como es el EEDP.

El objetivo del presente estudio, que forma parte de la validación nacional del ASQ, es determinar su correlación y concordancia con la escala EEDP. Además, se exploran las percepciones, experiencias y valoraciones comparativas entre ambos instrumentos, de acuerdo a profesionales que se encuentran aplicando dichos test.

\section{Pacientes y Métodos}

El presente estudio tiene un diseño de tipo cuanti- cualitativo. No experimental, transversal.

\section{Fase cuantitativa}

El Universo está conformado por lactantes de término aparentemente sanos, de nivel socioeconómico medio bajo, pertenecientes al primer y segundo quintil de ingreso, de acuerdo a la encuesta CASEN $2006^{17}$, que acudieron a control de supervisión de salud en 2 Centros de Salud de la Región Metropolitana, entre los meses de noviembre 2008 y diciembre 2009. El estudio fue aprobado por los comités de ética de los Centros de Salud involucrados.

La muestra fue de oportunidad, ya que incorporó lactantes que acudieron a su control sano y cuyos padres o cuidadores principales accedieron participar en el estudio. Se excluyeron los niños con diagnóstico confirmado de enfermedad neurológica, congénita o metabólica y los prematuros (menores de 37 semanas de edad gestacional).

Los cuidadores firmaron un consentimiento informado, completaron un formulario con datos de identificación, información sociodemográfica y de riesgo biológico del niño. Posteriormente completaron el cuestionario ASQ de la versión de 8 ó 18 meses según corresponda. En esos momentos, el profesional responsable del control de salud, que era ciego al resultado del ASQ, realizó el EEDP. Cada niño participó en una oportunidad del estudio. Los niños pesquisados con déficit en su DSM siguieron el algoritmo de derivación y estimulación establecido en los respectivos Centros de Salud.

\section{Instrumentos}

Ages and Stages Questionnaire third Edition $(A S Q-3)^{18}$

Es un cuestionario escrito de autoreporte para padres, que fue adaptado lingüísticamente para nuestro país previo al inicio del estudio. El instrumento está enfocado a evaluar el desarrollo en cinco áreas: comunicación, motor grueso, motor fino, solución de problemas y personal/social. Se consideró rendimiento deficitario si el niño evaluado tenía al menos en un dominio un puntaje $<-2$ DS del promedio.

\section{Escala de Evaluación del Desarrollo Psicomotor de 0-24 meses (EEDP) $)^{19}$}

Escala que evalúa el DSM en las aéreas motora, lenguaje, social y coordinación. El 
rendimiento general se expresa en un puntaje total estándar que se denomina Coeficiente de Desarrollo (CD). El rendimiento se considera deficitario cuando el CD es $<$ a $-1 D S$.

\section{Análisis estadístico}

Los datos obtenidos fueron recopilados en una planilla Excel y luego analizados con el programa estadístico Epi 6.

Se midió y comparó la frecuencia de déficit del DSM de acuerdo a ambos test, con el fin determinar la concordancia en el diagnóstico, considerando los casos evaluados. El criterio de interpretación del índice Kappa fue el siguiente: concordancia pobre: $0,00-0,20$; regular: 0,21-0,40; buena: 0,41-0,60; substancial $0,61-0,80$; casi perfecta o perfecta $0,81-1,00 .{ }^{15}$

Se midió la correlación mediante el coeficiente de Person, $\rho$ (rho), se catalogó como buenos los índices superiores a 0,7 y aceptables sobre 0,5 .

\section{Fase cualitativa}

Se realizaron entrevistas en profundidad individuales y mini focus groups, con el apoyo de una pauta guía semi estructurada, flexible y abierta que contenía los principales temas a investigar.

La selección de los entrevistados fue intencional de acuerdo a los criterios de inclusión, definidos en el grupo objetivo y perteneciente a consultorios participantes en la aplicación experimental de ASQ (tabla 1). La participación fue voluntaria, anónima y confidencial. Todas las personas entrevistadas, independientemente de su profesión, tenían experiencia en evaluación del DSM y en la aplicación de las escalas EEDP y ASQ.

\section{Procedimiento de análisis}

Los contenidos de cada entrevista se vaciaron en una matriz de análisis previamente estructurada de acuerdo a los objetivos, las categorías de análisis y los atributos en estudio y de acuerdo a los criterios de segmentación. Esto permitió ordenar, organizar, comparar, relacionar, comprender los contenidos y realizar el análisis sistemático de la información obtenida, extrayendo las principales tendencias, variables condicionantes de similitudes y diferencias observadas.

\section{Resultados}

La muestra del estudio fue conformada por 330 lactantes de nivel socio económico medio -bajo, atendidos en APS, que completaron ambas evaluaciones.

La prevalencia de déficit del DSM de acuerdo al EEDP fue $8,8 \%$ (IC 5,9-12,6) y según ASQ de 12,73\%, (IC 9,1-17,2), $\mathrm{p}=0,05$. Se encontró correlación aceptable entre ambas evaluaciones ( $\mathrm{r} 0,5, \mathrm{p}<0,01)$,) (figura 1). La concordancia en el diagnóstico de déficit de DSM fue buena, (kappa 0,576 IC 0,4- 0,7, p < $0,01)$, (tabla 2). No se encontraron diferencias significativas al desglosar la muestra por edad y por centro participante en el estudio.

\section{Fase cualitativa}

En las entrevistas a los profesionales que conocen y aplican ambos instrumentos, destacó su valoración positiva del ASQ, no sólo como instrumento de medición del DSM, sino que también como una guía para promover el DSM. Los principales aspectos mencionados al comparar el ASQ con el EEDP se presentan en las tablas 3 y 4.

Entre las fortalezas y ventajas comparativas del ASQ con respecto al test EEDP destaca:

- Es un test más moderno y más ajustado al nivel de desarrollo de los niños en la actualidad.

Tabla 1. Entrevistas y mini focus realizados

\begin{tabular}{|ccccc|}
\hline Técnica & $\begin{array}{c}\text { Profesionales con experiencia o conocimiento en ASQ Y EEDP } \\
\text { Entrevista en pro- } \\
\text { fundidad individual }\end{array}$ & Mini focus & Mini Focus & Total profesionales \\
\hline No y tipo de profesionales & 1 Médico familiar & 3 enfermeras & 2 parvularias & 6 \\
\hline
\end{tabular}






Figura 1. Correlación entre EEDP y ASQ.

Tabla 2. Concordancia entre EEDP y ASQ en la pesquisa de déficit del DSM

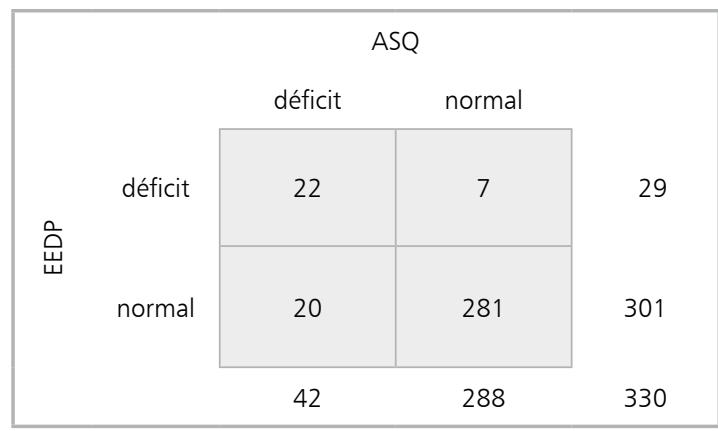

Kappa 0,576 (IC 0,4- 0,7)

- Es una escala más fina y completa, y, por lo tanto, más precisa para identificar los niños con déficit.

- El diagnóstico de desarrollo acorde al ASQ muestra mayor coincidencia con la observación clínica que el EEDP.

- Estimula la participación de los padres, otorgándoles un rol activo, a diferencia del EEDP que sitúa a los padres en una posición pasiva.

- La evaluación es más realista y acertada ya que mide al niño en su ambiente, en el contexto diario y a través del tiempo, en tanto el EEDP mide en un contexto extraño y en un momento particular.
- Despierta buena disposición de las madres y/o padres.

Las debilidades atribuidas al ASQ se relacionaron principalmente con su modalidad de autoreporte, especialmente al evaluar grupos de mayor riesgo socio cultural o educacional, ya que en esta modalidad, la evaluación depende directamente de los cuidadores.

- Hay una proporción significativa de padres o cuidadores que no poseen la preparación, capacidad de observación, comprensión lectora y compromiso necesario para completar en forma confiable el ASQ, lo que podría redundar en una menor pesquisa de un grupo de niños en riesgo.

- Se visualiza como un problema la falta de respuesta o devolución de los cuestionarios autoadministrados.

Algunos de los entrevistados asociaron una menor tasa de respuesta de los grupos de mayor riesgo social.

Para suplir las debilidades mencionadas, en los distintos centros de salud se adoptaron diferentes estrategias, como el dar un espacio adecuadamente implementado para completar el ASQ durante el tiempo de espera, hacer talleres grupales, o tener la posibilidad de com- 
Tabla 3. Valoración comparativa de los cuestionarios EEDP y ASQ en la evaluación del DSM

\begin{tabular}{|c|c|c|}
\hline Categorías & EEDP & ASQ \\
\hline $\begin{array}{l}\text { Características } \\
\text { generales }\end{array}$ & $\begin{array}{l}\text { Útil, simple, fácil de aplicar y rápido de tabular. } \\
\text { "Es fácil de aplicar, útil, rápido..., considerando la } \\
\text { cooperación del niño, claro" }\end{array}$ & $\begin{array}{l}\text { Muy buen instrumento, interesante, actual, eco- } \\
\text { nómico y capaz de discriminar déficit en distintas } \\
\text { áreas } \\
\text { Diseño llamativo, claro y simple de entender para } \\
\text { las mamás }\end{array}$ \\
\hline $\begin{array}{l}\text { Nivel de actualización } \\
\text { de la prueba }\end{array}$ & $\begin{array}{l}\text { Test antiguo que actualmente no se ajusta a la } \\
\text { realidad del desarrollo de los niños, especialmente } \\
\text { en el área de lenguaje. }\end{array}$ & $\begin{array}{l}\text { Es actual y acorde a la realidad de los niños de hoy. } \\
\text { "las conductas que están estipuladas son acordes } \\
\text { a la realidad" }\end{array}$ \\
\hline $\begin{array}{l}\text { Confiabilidad y validez } \\
\text { en la pesquisa }\end{array}$ & $\begin{array}{l}\text { No es preciso en la pesquisa de nivel de riesgo y } \\
\text { rezago } \\
\text { "Este test nos está dando un nivel de rezago que } \\
\text { es muy inferior a lo real" } \\
\text { La pesquisa se considera gruesa } \\
\text { Si bien detecta hitos fundamentales en las áreas } \\
\text { del DSM, deja fuera aspectos más finos que son } \\
\text { también importantes } \\
\text { "...muchos niños que caen en categoría normal, } \\
\text { pero nosotros definitivamente vemos que su desa- } \\
\text { rrollo no está bien" }\end{array}$ & $\begin{array}{l}\text { Si es adecuadamente completado, detecta tanto o } \\
\text { más que el EEDP los déficits del DSM } \\
\text { Brinda una evaluación más fina, y acuciosa de las } \\
\text { habilidades sicomotoras al poseer más sub áreas, } \\
\text { ser más extenso y más específico } \\
\text { Se aprecia mayor coincidencia entre sus resultados } \\
\text { y la impresión clínica que con el EEDP }\end{array}$ \\
\hline $\begin{array}{l}\text { Posibilidad de segui- } \\
\text { miento }\end{array}$ & No es un instrumento definido para el seguimiento & $\begin{array}{l}\text { Además de evaluar rezago, detecta el progreso en } \\
\text { las habilidades trabajadas }\end{array}$ \\
\hline $\begin{array}{l}\text { Reproducibilidad } \\
\text { (lugar de aplicación: } \\
\text { foto/realidad) }\end{array}$ & $\begin{array}{l}\text { Evalúa el desempeño del momento, el cual se ve } \\
\text { altamente influido por la situación puntual } \\
\text { "...se pasan cosas, porque es una foto imprecisa } \\
\text { del momento" }\end{array}$ & $\begin{array}{l}\text { Evalúa al niño en su hogar, lo que elimina los ses- } \\
\text { gos de ser evaluado en un ambiente ajeno y por } \\
\text { personas extrañas }\end{array}$ \\
\hline $\begin{array}{l}\text { Aplicabilidad en Aten- } \\
\text { ción Primaria }\end{array}$ & $\begin{array}{l}\text { Instrumento conocido y habitual y por lo tanto sig- } \\
\text { nifica ahorro de trabajo, de tiempo y de esfuerzo }\end{array}$ & $\begin{array}{l}\text { Necesidad de capacitación para su aplicación } \\
\text { masiva }\end{array}$ \\
\hline
\end{tabular}

Tabla 4. Valoración comparativa de los cuestionarios EEDP y ASQ en relación al impacto familiar

\begin{tabular}{|c|c|c|}
\hline $\begin{array}{l}\text { Involucramiento } \\
\text { padres }\end{array}$ & $\begin{array}{l}\text { La madre adopta un rol pasivo en un ámbito don- } \\
\text { de lo ideal sería que su participación fuera muy } \\
\text { activa y protagónica } \\
\text { "...el problema es el desinterés de la mamá. La } \\
\text { falta de compromiso de la familia" } \\
\text { "A pesar de que das indicaciones, muchas veces } \\
\text { vuelven después de unos meses y los resultados } \\
\text { son los mismos, no ha habido cambio por falta de } \\
\text { cooperación" }\end{array}$ & $\begin{array}{l}\text { Su modalidad de auto reporte es más amigable } \\
\text { y tiene la virtud de validar a la madre o familiar } \\
\text { a cargo: } \\
\text { "con este test son las madres las protagonistas y } \\
\text { lo lindo es que se dan cuenta de lo que hay que } \\
\text { trabajar" } \\
\text { Permite el empoderamiento de los padres } \\
\text { Los padres o cuidadores a cargo aprenden a } \\
\text { observar el desarrollo de sus hijos y se fortalece } \\
\text { el vínculo } \\
\text { "implica un abrir de ojos de los padres en relación } \\
\text { al desarrollo de los niños" }\end{array}$ \\
\hline $\begin{array}{l}\text { Reacciones de las } \\
\text { madres frente al } \\
\text { diagnóstico de déficit } \\
\text { de DSM }\end{array}$ & $\begin{array}{l}\text { Predomina la indiferencia y falta de compromiso } \\
\text { y comprensión } \\
\text { "hay mamás que no lo creen y dicen -no, pero } \\
\text { cómo, si mi hijo lo hace todo" }\end{array}$ & $\begin{array}{l}\text { La aplicación del test despierta una actitud pro- } \\
\text { activa frente al desarrollo psicomotor normal y } \\
\text { posibles deficiencias } \\
\text { "me dicen... sabe que señorita, tal cosa no la hizo } \\
\text { pero me gustaría saber cómo puedo hacer para } \\
\text { que lo haga" }\end{array}$ \\
\hline $\begin{array}{l}\text { Efecto estimulador en } \\
\text { la familia y fortaleci- } \\
\text { miento del vínculo }\end{array}$ & $\begin{array}{l}\text { Permite motivar y entrenar a la madre sobre el } \\
\text { DSM del niño de acuerdo a su edad }\end{array}$ & $\begin{array}{l}\text { Constituye un instrumento no sólo de evaluación } \\
\text { sino también una guía de estimulación para el } \\
\text { niño y de motivación a la participación familiar } \\
\text { Padres asumen responsabilidad en la estimulación } \\
\text { de los hijos }\end{array}$ \\
\hline
\end{tabular}


pletar el ASQ con asistencia profesional.

En el momento de optar por uno de los dos instrumentos para la APS, los entrevistados propusieron el uso del ASQ como un complemento del EEDP.

\section{Discusión}

Este estudio, que forma parte de un proyecto de validación nacional e implementación de ASQ, se diseñó para conocer si este instrumento tendría ventajas sobre las pruebas actualmente disponibles en APS.

Al comparar los resultados obtenidos en el ASQ y el EEDP, destaca la correlación aceptable y buena concordancia entre ambas evaluaciones, lo que sería esperable dado que ambos cuestionarios cumplen el mismo propósito y miden aspectos similares del DSM.

A pesar que la frecuencia de pesquisa de dificultades del DSM fue significativamente superior acorde al ASQ que al EEDP, acercándose a estimaciones internacionales ${ }^{5,6}$, llama la atención que fue inferior a reportes del uso de ASQ en otros sectores de la población y en otros países, en que la prevalencia reportada se aproxima al 20\% ${ }^{11,13,20-22}$.

Al igual que lo muestran los datos cuantitativos, en el análisis cualitativo se refirió que el ASQ sería un instrumento más exigente que el EEDP en la pesquisa de déficits del DSM; seguramente en este aspecto influye que mientras que en EEDP el diagnóstico se basa en el puntaje total, el ASQ considera cada área por separado.

Entre los principales aportes del ASQ, se destacó también el involucramiento de los padres en la observación del desarrollo de sus hijos, aspecto que sin duda puede tener un efecto multiplicador de las acciones preventivas en salud.

Pese a las ventajas comparativas del ASQ, los profesionales manifestaron algún grado de aprehensión de relacionado con la modalidad de autoreporte, aspecto que podría afectar una pesquisa confiable de rezagos en poblaciones de mayor vulnerabilidad social. Al respecto cabe destacar que estudios realizados en diferentes países y culturas han mostrado que el uso de cuestionarios de autoreporte parental son altamente sensibles ${ }^{23-25}$ independiente del nivel educacional, socioeconómico y ubicación geográfica de los cuidadores ${ }^{26-28}$.

En los centros de salud que participaron del estudio, se adoptaron estrategias para reducir el efecto del riesgo socio-cultural y capacidad intelectual de participar en la modalidad de autoreporte, como dar un espacio especial para completar el cuestionario en la sala de espera, hacer talleres grupales y ofrecer asesoría para completar ASQ. Creemos que es necesaria mayor investigación para comprender el impacto de dichas estrategias para el trabajo con familias de mayor vulnerabilidad.

Finalmente, debemos reflexionar sobre nuestra capacidad de implementar una medición de autoreporte como método de screening del desarrollo infantil en APS. En el momento de optar por una de las evaluaciones, los entrevistados propusieron un periodo de transición, mientras se diseñan estrategias que permitan una adecuada capacitación e implementación del ASQ, de acuerdo a cada realidad local.

Indudablemente, a nivel país debemos actuar sobre las brechas socioeconómicas que persisten en la salud y el desarrollo infantil. Para ello es imperativo, no sólo intervenir sobre los determinantes de la salud, sino que además es fundamental la actualización e implementación de herramienta objetivas, que mejoren la pesquisa de los niños que se encuentran con déficit e involucrar más a los padres en su pesquisa y estimulación. Este estudio muestra que el ASQ puede ser una de estas herramientas.

\section{Referencias}

1.- Council on Children with Disabilities, Section on Developmental Behavioral Pediatrics, Bright Futures Steering Committee and Medical Home Initiatives for Children with Special Needs Project Advisory Committee: Identifying Infants And Young Children With Developmental Disorders In The Medical Home: An Algorithm for Developmental Surveillance and Screening. Pediatrics 2006; 118: 405-20.

2.- Rodríguez $S$, Arancibia V, Undurraga $C$ : Escala de evaluación del Desarrollo Psicomotor de 0 a 24 meses. Editorial Galdoc, Santiago de Chile, 1985. 
3.- Normas técnicas de evaluación y estimulación del desarrollo psicomotor en el niño y la niña menor de 6 años. Ministerio de Salud. Santiago Chile 2004. [accedido 26 de mayo 2007] disponible en http://www.minsal.cl/ ici/S_1/salud_nino/Manual.pdf

4.- Ministerio de Salud de Chile: El futuro de los niños es siempre hoy. Propuestas del Consejo Asesor Presidencial para la Reforma de las Políticas de Infancia. 2006.

5.- Committee of children with disabilities: Developmental surveillance and screening of infant and young children. Pediatrics 2001;108 (1): 192-5.

6.- Boyle CA, Decoufle P, Yeargin-Allsoop MY: Prevalence and health impact of developmental disabilities. Pediatrics 1994; 93:863-5.

7.- Schonhaut L, Maggiolo M, De Barbieri Z, Rojas P, Salgado A: Dificultades de lenguaje en preescolares: concordancia entre el test tepsi y la evaluación fonoaudiológica. Rev Chil Pediatr 2007; 78 (4): 369-75.

8.- Bedregal P, Scharager J, Breinbauer C: El desarrollo de un modelo para la evaluación de rezagos del desarrollo infantil de Chile. Rev Med Chile 2007; 135 (3): 403-5.

9.- Subsecretaría de Salud Pública División de Planificación Sanitaria: II Encuesta de Calidad de Vida y Salud, Chile 2006. Disponible en: http://epi.minsal.cl/epi/html/ sdesalud/calidaddevida $2006 /$ Informe $\% 20$ Final $\% 20$ Encuesta $\% 20$ de $\% 20$ Calidad $\% 20$ de $\% 20$ Vida $\% 20 y \% 20$ Salud\%202006.pdf

10.- Rydz D, Srour M, Oskoui M, Marget $N$, et al: Screening for Developmental Delay in the Setting of a Community Pediatric Clinic: A Prospective Assessment of Parent-Report Questionnaires Pediatrics 2006; 118 : 1178-86.

11.- Sices L, Drotar D, Keilman A, H. Kirchner L, Roberts $D$, Stancin T: Communication About Child Development During Well-Child Visits: Impact of Parents' Evaluation of Developmental Status Screener With or Without an Informational Video. Pediatrics 2008; 122: e1091-9.

12.- Squires J, Bricker D, Potter LW: Revision of a ParentCompleted Developmental Screening Tool: Ages and Stages Questionnaires. Journal of Pediatric Psychology 1997; 22 (3): 313-28.

13.- Eun Young Kim, In Kyung Sung: The Ages and Stages Questionnaires: screening for developmental delay in the setting of a pediatric outpatient clinic. Korean $\mathrm{J}$ Pediatr 2007; 50 (11): 1061-6.

14.- Agnes Tsai H, Mcclelland M, Pratt C, Squires J: Adaptation of the 36 month age Ages and Stages Questionnaires in Taiwan: Results from preliminary study. J
Early Intervent 2006; 28 (3): 213-25.

15.- Richter J, Harald J: A validation study of the Norwegian version of the Ages and Stages Questionnaires. Acta Paediatr 2007; 96: 748-52.

16.- Schonhaut L, Salinas P, Armijo I, Schonstedt M, Álvarez $J$, Manriquez M: Screening de desarrollo psicomotor en la consulta pediátrica: evaluación del una prueba de auto-reporte de los padres. Rev Chil Pediatr 2009; 80 (6): 513-9.

17.- www.mideplan.cl

18.- Squires J, Bricker D: Ages \& Stages Questionnaires[R], Third Edition (ASQ-3[TM]): A Parent-Completed Child-Monitoring System. Brookes Publishing Company. Baltimore, USA 2008.

19.- Rodríguez S, Arancibia V, Undurraga C: Escala de evaluación del Desarrollo Psicomotor de 0 a 24 meses. Editorial Galdoc, Santiago de Chile, 1985.

20.- Yu LM, Hey E, Doyle LW, Farrell B, Spark P, Altman DG, Duley L; Magpie Trial Follow-Up Study Collaborative Group: Evaluation of the Ages and Stages Questionnaires in identifying children with neurosensory disability in the Magpie Trial follow-up study. Acta Paediatr 2007; 96 (12): 1803-8.

21.- Hix-Smallh, Marks K, Squires J, Nickel R: Impact of Implementing Developmental Screening at 12 and 24 Months in a Pediatric Practice. Pediatrics 2007; 120 (2): 381-9.

22.- Schonhaut L, Schonstedt M, Álvarez J, Salinas P, Armijo I: Desarrollo psicomotor en niños de nivel socioeconómico medio alto. Rev Chil Pediatr 2010; 8 (2): 122-7.

23.- Bailey D, Hebbeler K, Scarborough A, Spiker D, Mallik $S$ : First Experiences With Early Intervention: A National Perspective. Pediatrics 2004; 113 (4): 887-96.

24.- ASQ: Technical report, descargado desde http://www. brookespublishing.com/store/books/bricker-asq/index. htm el 14 de agosto de 2007.

25.- Regalado M, Halfon N: Primary Care Services promoting optimal child development from birth to age 3 years. Arch Pediatr Adolesc Med 155: 1311-22.

26.- Knobloch H, Stevens F, Malone A, Ellison P, Risemberg $H$ : The validity of parental reporting of infant development. Pediatrics 1979; 63: 872-8.

27.- Glascoe FP, Dworkin PH: The role of parents in the detection of developmental and behavioral problems. Pediatrics 1995; 95: 829-36.

28.- Squires J, Potter L, Bricker D And Lamorey S: Parentcompleted developmental Questionnaires: Effectiveness with low and middle income parents. Early Child Res Quart 1998; 13 (2): 345-54. 